NANOSCIENCE AND TECHNOLOGY- AN INTERNATIONAL JOURNAL

\author{
ISSN Print: 2572-4258 \\ ISSN Online: 2572-4266 \\ PUBLISHER - BEGELL HOUSE (USA) \\ Accepted February $11^{\text {th }} 2020$

\section{A REVIEW ON RECENT ADVANCEMENTS IN THE HEMODYNAMICS OF NANO-DRUG DELIVERY SYSTEMS}

\author{
Jayati Tripathi' ${ }^{1}$ B. Vasu ${ }^{1}$, Rama S R Gorla ${ }^{2}$, P. V. S. N. Murthy ${ }^{3}$, O. Anwar Bég ${ }^{4}$ and P. Saikrishnan ${ }^{5}$ \\ ${ }^{1}$ Department of Mathematics, Motilal Nehru National Institute of Technology Allahabad, Prayagraj- \\ 211004, India, Email: jayati@mnnit.ac.in, bvasu@mnnit.ac.in \\ ${ }^{2}$ Department of Mechanical Engineering, Cleveland State University, Ohio, USA \\ ${ }^{3}$ Department of Mathematics, Indian Institute of Technology Kharagpur, India \\ ${ }^{4}$ Department of Mechanical Engineering, SEE, Salford University, Salford, M54WT, UK \\ ${ }^{5}$ Department of Mathematics, National Institute of Technology, Trichy- 620015, India.
}

\begin{abstract}
:
Cardiovascular disease (CVD) is a leading cause of mortality and morbidity in developed countries. CVD is produced by atherosclerotic lesions that reduce arterial lumen size through plaque formation and arterial thickening. This decreases blood flow to the heart and frequently manifests in severe hemodynamic complications like myocardial infarction or angina pectoris. A drug delivery system (DDS) is a clinical methodology (formulation or device) which enables the introduction of a therapeutic substance into the body and improves its efficacy and safety by controlling the rate, time, and place of release of drugs in the body. Drug delivery technologies modify drug release profile, absorption, distribution and elimination for the benefit of improving product effectiveness and patient convenience and compliance. The review explores extensively hemodynamic aspects of the cardiovascular system and diseases which can be treated via nanodrug delivery with a comprehensive overview of research efforts in these areas. Nanomedicine is an expeditiously growing science in which biomaterials (drugs) engineered at the nanoscale are implemented to enhance therapeutic performance and improve patient treatments. Among the many other diverse applications of nanomaterials in medicine (e.g. bio-UIRtribology, tissue repair, orthopaedic implants etc), nano-drug delivery systems have emerged as among the most promising. This technology has evolved into a significant platform for delivering successfully remedial agents to diseased sites with substantially greater target control, precision and sophistication. By greatly increasing site specificity, lowering toxicity and target-oriented
\end{abstract}


delivery, nanotechnological drug delivery ("nano-pharmacodynamics") has consistently achieved very impressive consistency, benefits and has aided massively in the fight against potentially lethal haemotological diseases. Recently, nanomedicine has embraced an even wider range of applications including the administration of chemotherapeutic agents, biological agents, diabetes regulation, sterilization, cancer and tumour inhibition, rheumatic fever mitigation etc. The current review presents a comprehensive appraisal of nano-drug delivery systems, simulation with engineering methods, types of nanodrugs and their effectiveness. The excellent targeting properties attainable with magnetic nanoparticles as engineering pharmacodynamic agents, in particular, offers huge potential in the treatment of many complex hemodynamic disorders. Furthermore, the present review summarizes the efficiency of drug carrier nanoparticles in mitigating the adverse effects of stenosed blood vessels and outlines other future potential uses for nano-drugs in biomedical applications.

Keywords: Drug Delivery System; Nanoparticles; Blood Flow; Cardiovascular Diseases; NanoMagnetic Drug Delivery; Nanomedicine; Arterial Lumen.

1. Introduction

1.1 Clinical Research Importance

1.2 Nanofluidics

1.2.1 Thermophysical Properties

1.2.2 Application

2. Drug Delivery Systems

2.1 Drug Designing and Drug Delivery Process

2.2 Nano-based Drug Delivery Systems

2.3 Nano-Drug Designing and Nano-Drug Delivery Process

2.4 Nanoparticles Used in Drug Delivery

2.5 Nano-Magnetic Drug Delivery

3. Nano-based Drug Delivery for Cardiovascular Diseases

3.1 Significance

3.2 Nano-based Blood Flow (Nano-Hemodynamic) Models

4. Conclusions

References

\section{Introduction}

Hemodynamics has an integral role in the formation and evolution of cardiovascular diseases. Accumulation of macrophage white blood cells, low-density lipoproteins (LDL) and deposition of 
cholesterol to the arterial wall of blood vessels results in a hardening of the arteries and reduction in the cross-sectional area of the blood vessel thereby leading to cardiovascular diseases. Simulation of blood flow has been widely used in recent decades for better understanding the symptomatic spectrum of various diseases, in order to improve already existing clinical treatments or to develop new therapeutic techniques. The characteristics of the blood flow in an artery can be changed significantly by arterial disease and important examples of geometric modifications to the arterial structure are aneurysms and stenoses. Blood vessels are tapered by the growth of atherosclerotic plaques that bulge into the lumen, resulting in stenosed blood vessels. Thus, the development of a stenosis can lead to severe circulatory and arterial disorders. In the recent past a considerable number of theoretical and experimental investigations related to blood flow in arteries in the presence of a stenosis have been reported with various perspectives in the area of arterial biomechanics depending upon the objectivity of the problems of the life sciences. Human blood is a heterogeneous multiphase suspension of blood cells (red blood, white blood, platelets) in plasma, which constitutes about $55 \%$ of total blood volume and is composed of mostly water, dissipated proteins, mineral ions, clotting factors, hormones and blood cells [1]. It has been pointed out that the plasma behaves as a Newtonian fluid [2]; however, whole blood exhibits non-Newtonian fluid nature [3]. For shear rate larger than $100 \mathrm{~s}^{-1}$, blood exhibits a Newtonian nature, and this arises in for example, large arteries, veins and in large cavities. However, for shear rate less than $100 \mathrm{~s}^{-1}$, blood behaviour is non-Newtonian i.e. hemo-rheological.

\begin{tabular}{|c|c|c|c|c|}
\hline \multicolumn{2}{|l|}{ Blood component } & Per microliter & Size $(\mu \mathrm{m})$ & Percentage \\
\hline Red blood cell & & $4.1-5.1 \times 10^{6}$ & $7-8$ & 97 \\
\hline $\begin{array}{l}\text { White blood cell } \\
\left(4-10 \times 10^{3}\right)\end{array}$ & $\begin{array}{l}\text { Neutrophils } \\
\text { Lymphocytes } \\
\text { Eosinophils } \\
\text { Monocytes } \\
\text { Basophils }\end{array}$ & $\begin{array}{l}62 \% \text { of } \mathrm{WBC} \\
30 \% \text { of } \mathrm{WBC} \\
2.3 \% \text { of } \mathrm{WBC} \\
5.3 \% \text { of } \mathrm{WBC} \\
0.4 \% \text { of } \mathrm{WBC}\end{array}$ & $\begin{array}{l}10-12 \\
6-14 \\
- \\
15-20 \\
-\end{array}$ & 2 \\
\hline Platelet & & $1.5-4.5 \times 10^{5}$ & 3 & 1 \\
\hline
\end{tabular}

Table 1: Blood Cell Characteristics [1] 
Across a wide range of cardiovascular diseases, the best outcome depends on the ability to successfully direct drugs towards a specific diseased zone. Despite intense research and developments in drug delivery, present-day pharmacological formulations still leave the drug incapable of precisely localizing en mass at sites of interest. The drug molecules diffuse and spread in many cases randomly throughout the body, resulting in undesirable side effects and a reduction in the active response of proper doses. In recent years a new branch of fluid dynamics, focused on engineering fluids at the nanoscale, has emerged - nanofluid dynamics. Nanofluids have had a significant impact in improving miscellaneous applications in energy systems, industrial processes, transport, environmental and biomedical sciences.

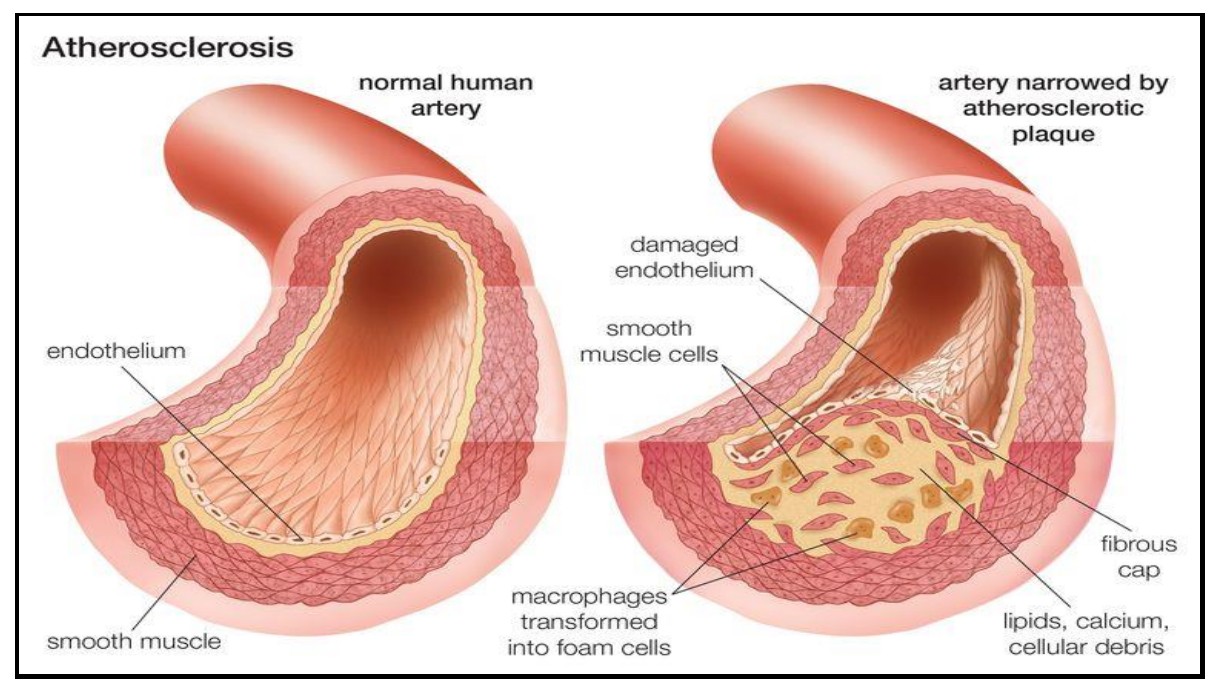

Figure 1: A normal artery versus diseased artery [88]

\subsection{Clinical Research Importance}

According to World health organization (WHO) report 2017 [4], Cardiovascular diseases (CVDs) are the first cause of deaths worldwide. Annually more people die because of CVDs in comparison with any other diseases. In 2016, almost 17.9 million deaths were documented due to CVDs, which corresponds to approximately $31 \%$ of total deaths. In addition, $85 \%$ of these deaths are due to strokes and heart attacks. The WHO report also emphasizes that low and middle-income countries are more prone to cardiovascular diseases as almost three quarters of total CVDs deaths arose in these countries. Out of 17 million premature deaths attributable to non-communicable diseases in $2015,37 \%$ are due to CVDs and $82 \%$ are in low and middle-income countries. 


\begin{tabular}{|c|c|c|c|}
\hline & \multicolumn{3}{|c|}{ Percentage of total deaths ( $95 \%$ UI) } \\
\hline & Both sexes & Men & Women \\
\hline Cardiovascular diseases & $28 \cdot 1 \%(26 \cdot 5-29 \cdot 1)$ & $29 \cdot 2 \%(27 \cdot 5-30 \cdot 3)$ & $26 \cdot 7 \%(23 \cdot 8-28 \cdot 3)$ \\
\hline Ischaemic heart disease & $17 \cdot 8 \%(16-8-18 \cdot 5)$ & $19 \cdot 6 \%(18 \cdot 5-20-4)$ & $15 \cdot 6 \%(13 \cdot 9-16 \cdot 6)$ \\
\hline Stroke & $7 \cdot 1 \%(6 \cdot 6-7 \cdot 5)$ & $6 \cdot 9 \%(6 \cdot 4-7 \cdot 3)$ & $7 \cdot 3 \%(6 \cdot 5-7 \cdot 9)$ \\
\hline Hypertensive heart disease & $1 \cdot 3 \%(1 \cdot 1-1 \cdot 5)$ & $1.1 \%(0.9-1.4)$ & $1 \cdot 6 \%(1 \cdot 2-1 \cdot 9)$ \\
\hline Rheumatic heart disease & $1 \cdot 1 \%(1 \cdot 0-1 \cdot 2)$ & $0.8 \%(0.7-0.9)$ & $1.5 \%(1 \cdot 3-1 \cdot 7)$ \\
\hline Atrial fibrillation and flutter & $0.21 \%(0.16-0.26)$ & $0.17 \%(0.13-0.21)$ & $0.25 \%(0.20-0.32)$ \\
\hline Aortic aneurysm & $0-15 \%(0-14-0 \cdot 17)$ & $0.20 \%(0.18-0.21)$ & $0.10 \%(0.09-0.11)$ \\
\hline $\begin{array}{l}\text { Other cardiovascular and } \\
\text { circulatory diseases }\end{array}$ & $0.14 \%(0.09-0.17)$ & $0.13 \%(0.07-0.18)$ & $0.15 \%(0.08-0.18)$ \\
\hline Cardiomyopathy and myocarditis & $0.12 \%(0.09-0.13)$ & $0.12 \%(0.09-0.15)$ & $0.11 \%(0-07-0.13)$ \\
\hline Endocarditis & $0.12 \%(0.10-0.15)$ & $0.11 \%(0.09-0.16)$ & $0.14 \%(0.11-0.18)$ \\
\hline Peripheral artery disease & $0.01 \%(0.07-0.03)$ & $0.02 \%(0.01-0.04)$ & $0.01 \%(0.01-0.02)$ \\
\hline
\end{tabular}

Figure 2: \% of total deaths due to each aspect of cardiovascular diseases in India, 2016 [5] In 2016, cardiovascular diseases contributed $28.1 \%$ of the total deaths in India. For CVDs, the dominating risk factors include high systolic blood pressure, air pollution, high cholesterol, dietary risks, tobacco use and high body mass index. The main causes under cardiovascular diseases with the percentage of death rates are presented in Figure 2.

\subsection{Nanofluids}

Conventional fluids (air, water, oils etc) in industrial applications exhibit excellent lubrication properties; however, they have poor thermal characteristics which severely restrain their use. Nowadays, many researchers have been focused on developing techniques to increase heat transfer rates of conventional fluids. Experimentally it was found that the addition of small-sized solid particles in the base fluid can greatly enhance the thermo-physical properties. In 1995, Choi [6] coined a new term "nanofluid", which is defined as a colloidal suspension of nanometer-sized particles (1-100nm) in a base fluid. These nanoparticles can be oxide, carbide, metallic, nonmetallic, carbonic, hybrid and even more liquid droplets. The base fluid includes water, mineral oil, ethylene glycol or refrigerants. It has been shown that the inclusion of nanoparticles (termed "doping") in base fluids increases the thermal conductivity remarkably and this contributes principally to the improved thermal performance of nanofluids. The nanoparticles employed in biomedicine are also synthetic due to their unique interaction with biological matter $[7,8]$. 


\subsubsection{Thermophysical Properties}

In comparison with base fluids, nanofluids are formed have significantly different thermophysical properties such as thermal conductivity, viscosity, density and specific heat capacity.

(A) Density: With the help of the properties of both base fluid and nanoparticles, the effective density of nanofluid is numerically calculated by [9] as:

$$
\rho_{n f}=(1-\varphi) \rho_{f}+\varphi \rho_{s}
$$

Where $\rho_{f}$ and $\rho_{s}$ is a density of the base fluid and nanoparticle respectively and $\varphi$ is nanoparticle volume fraction.

(B) Specific Heat Capacity: Effective heat capacity can be computed using the following equation as reported in [9] as-

$$
\left(C_{p}\right)_{n f}=(1-\varphi)\left(C_{p}\right)_{f}+\varphi\left(C_{P}\right)_{s}
$$

Some other approaches have also been suggested regarding the heat capacity concept [10], for example-

$$
\left(\rho C_{p}\right)_{n f}=(1-\varphi)\left(\rho C_{p}\right)_{f}+\varphi\left(\rho C_{P}\right)_{s}
$$

(C) Viscosity: For the effective viscosity of a nanofluid, many models have been suggested by researchers considering the nanoparticle suspension in the base fluid. The first model for a suspension was suggested by Einstein [11] in 1906 and the derived equation is:

$$
\mu_{n f}=(1+2.5 \varphi) \mu_{f}
$$

As the model given by Einstein is restricted to spherical particles only, some other models also have been proposed by many researchers for non-spherical particles. A very important model was proposed by Brinkman [12] in 1952, which is-

$$
\mu_{n f}=\frac{\mu_{f}}{(1-\varphi)^{2.5}}
$$

(D) Thermal Conductivity: Hamilton and Crosser [13] presented a robust model for the thermal conductivity of solid-liquid mixtures of non-spherical particles, which is given below as:

$$
\frac{k_{n f}}{k_{f}}=\frac{k_{s}+(n-1) k_{f}-(n-1) \varphi\left(k_{f}-k_{s}\right)}{k_{s}+(n-1) k_{f}+\varphi\left(k_{f}-k_{s}\right)}
$$

Where $k_{s}$ and $k_{f}$ are the thermal conductivity of nanoparticles and base fluid, respectively, $n$ is an empirical shape factor, calculated as $n=\frac{3}{\psi}$ in which $\psi$ is the particle sphericity. 


\subsubsection{Application}

Generally, the rate of heat exchange in thermal systems is enhanced by using a nanofluid, due to the superior thermal conductivity over the conventional fluid. Therefore, by using nanofluids to enhance the heat transfer rate, the size of a thermal system can be optimized by transferring a specific value of heat which makes the system more compact. Relative to microfluids, nanofluids possess a higher stability and better potential to increase the heat conduction [14]. In many heating and cooling applications, nanofluids are superseding conventional fluids. Nanofluids can be used in car radiators, boilers, cooling of electronic equipment, solar collector systems, refrigerators, pharmacological drug delivery, sterilization, fuel cells, lubrication of components, aerospace coatings and fuels, enhanced oil recovery techniques and liquid based heat exchangers. Figure 3 gives an overview of different applications of nanofluids.

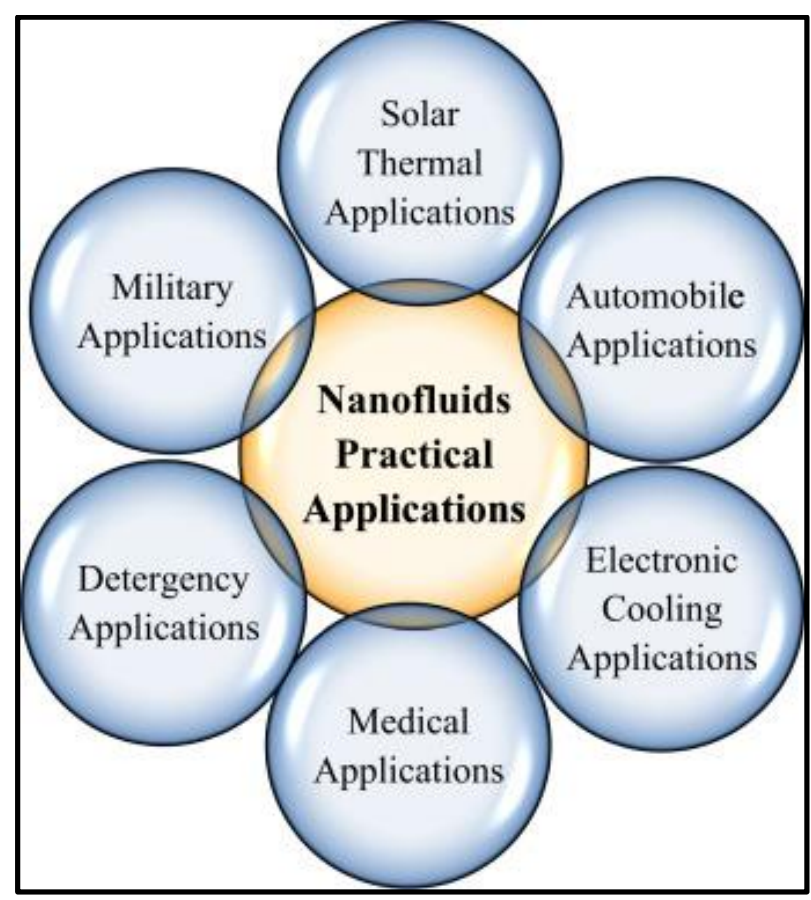

Figure 3: Applications of Nanofluids [15]

\section{Drug Delivery Systems}

\subsection{Drug Designing and Drug Delivery Process}

For many years, it has been found that the delivery of therapeutic compound to a target area is a primary obstacle in the treatment of several diseases. Limited effectiveness, poor bioavailability and solubility and also toxicity are major disadvantages of traditional drugs. To overcome these 
limitations and drawbacks, controlled drug delivery has been proposed and developed. For drug delivery, there are essentially two aims: firstly, to reduce the drug side effects by specifically targeting the desired organ and secondly to avoid overdosing or under-dosing cycles by controlled release of the drug [16]. A drug delivery system (DDS) is a formulation or a device that enables the introduction of a therapeutic substance into the body and improves its efficacy and safety by controlling the rate, time, and place of release of drugs in the body. In particular, a DDS constitutes precision-engineered biotechnology for the controlled release and/or targeted delivery of therapeutic handler. Controlled drug delivery systems are designed in a way that the drug is transported to the target region with simultaneous avoidance (or at least reduction) in any undesired influence on vital tissues and unwanted side effects [17]. This delivery system defends the drug from expeditious deterioration and increases the drug concentration in targeted tissues, which results in the benefit of requiring lower doses of drugs. This therapy is found to be important when there is variation between a dose of drug and its toxic effects in body. To further improve therapeutic effects and reduce the undesirable side (toxic) effects, continuous development and refinement of suitable drug vehicles is a growing field in biomedical and pharmaceutical engineering.

\subsection{Nano-based Drug Delivery Systems}

In drug delivery systems, using large size of materials or carriers poses major challenges including poor bioavailability and solubility, issue with target specificity, in vivo stability and tonic effectiveness with probable side effects. Therefore, in advanced biomedicine, nanotechnology offers substantial promise in drug formulations which control drug release and deliver the drug with more specificity. Nanomaterials are materials engineered in the range between 1 and $100 \mathrm{~nm}$. Nanoparticles have been extensively implemented in recent years in many sub-branches of biomedicine including drug delivery, tissue engineering and biosensors [18, 19]. Nanoparticles are usually small sized nanospheres; hence in comparison to larger particles they can move more freely in the human body. Nowadays nanomedicine has matured into a major area of biomedicine since nano-designed delivery agents can be embedded in therapeutic drugs and targeted drug delivery can be achieved with controlled release [20]. The first generation of nanoparticle-based therapy has been based on lipids systems like micelles and liposomes [21], which can contain inorganic nanoparticles like silver, gold or metallic. Nanostructures stay in the circulatory system for 
prolonged duration and allow the release of drugs at fixed doses and rates, which cause less plasma fluctuations leading to minimized side effects [22].

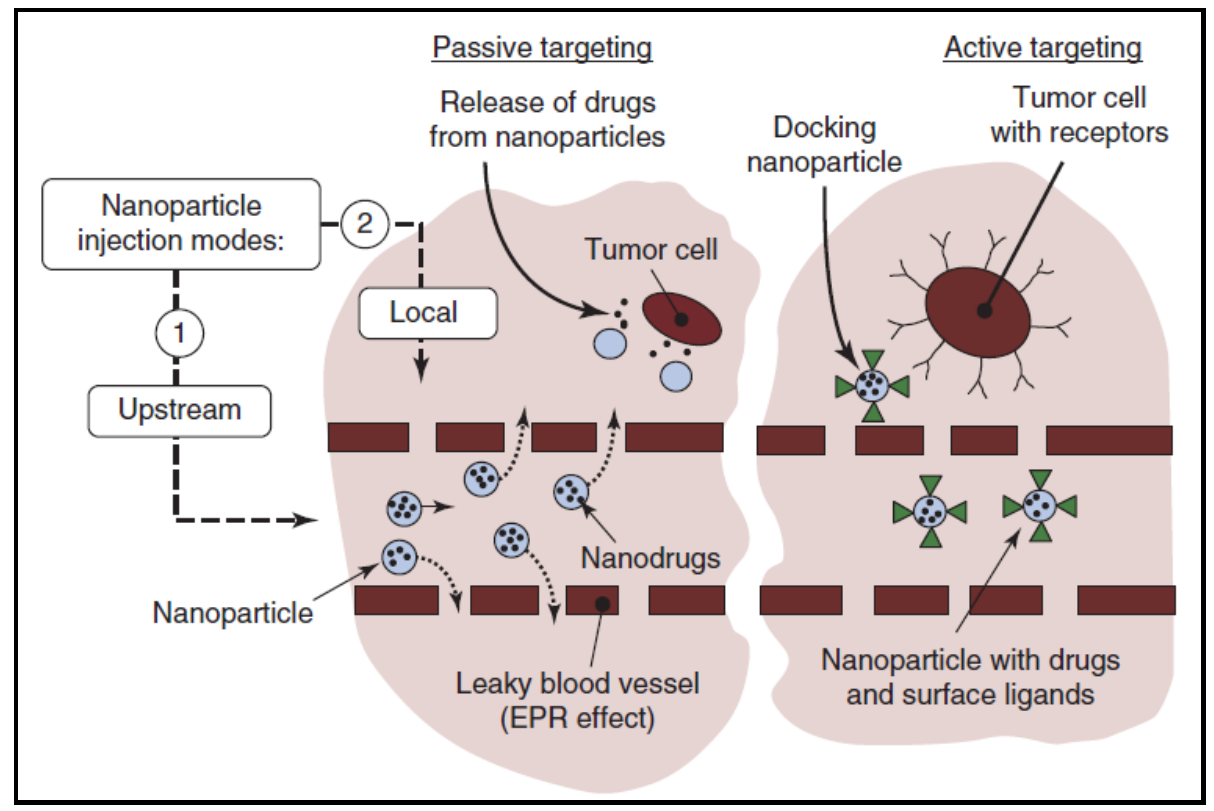

Figure 4: Passive targeting vs Active targeting [23]

In drug delivery using nanomaterials the selection of a particular nanoparticle, is based, on the desired physical and chemical properties of the intended nano-drugs. Two mechanisms are known via which nano-engineered pharmacological agents deliver: self-delivery and passive. In the latter, drugs are encapsulated in the inner cavity of nanostructure and when the nanostructured drug is targeted to a particular region the required amount of drug is then released. In the former, the drugs required to be released are directly attached to the carrier nanostructure for easy delivery. In the self-delivery approach, the timing of release is a crucial aspect since failure in this regard may result in the drug not reaching the targeted site [24]. In drug delivery systems, targeting of drugs using nano formulation is also classified into active and passive targeting. In passive targeting, the prepared drug carrier circulates in the blood flow and is driven to the targeted site by binding which is strongly influenced by properties like temperature, $\mathrm{pH}$, molecular shape and nanoparticle dimensions. In active targeting, antibodies and peptides are coupled with systems to the surface of the nanocarriers by different conjugation chemistries. Therefore, nanotechnology provides various benefits in treating human diseases with improved site specificity of drugs and more precise targeted delivery of medicines. 


\subsubsection{Nano-Drug Designing and Nano-Drug Delivery Process}

With the progression of drug delivery systems and design, to boost the drug specificity and diagnostic accuracy, various conventional methods have been reviewed and many therapeutic procedures have been suggested. In light of this, new robust ways of administrating the drug have been rigorously examined, which are focused on reducing the side effects by increasing their bioavailability in the organ so that controlled or targeted delivery, can be achieved [25]. With reference to this, drug design is a critical aspect that characterizes the compatibility of the principal drug in the context of a specific biological target. Several studies have been found related to this which focuses on the design of different particles and the importance of distinctive mechanisms of drug release [26]. Each drug delivery system has its own physical, morphological and physical properties and possesses unique compatibility for various drugs polarities through physical and chemical interactions. For understanding drug delivery profiles, an appreciation of some other parameters including the compositions of nanocarriers (e.g. inorganic, organic etc) and the state in which drugs are mixed with them (matrix system or core shell system) is also essential [27]. Release of drugs in nanocarriers is represented by different mechanisms which include diffusion, stimuli-sensitive, solvent and chemical reaction [28, 29], as depicted in figure 2.

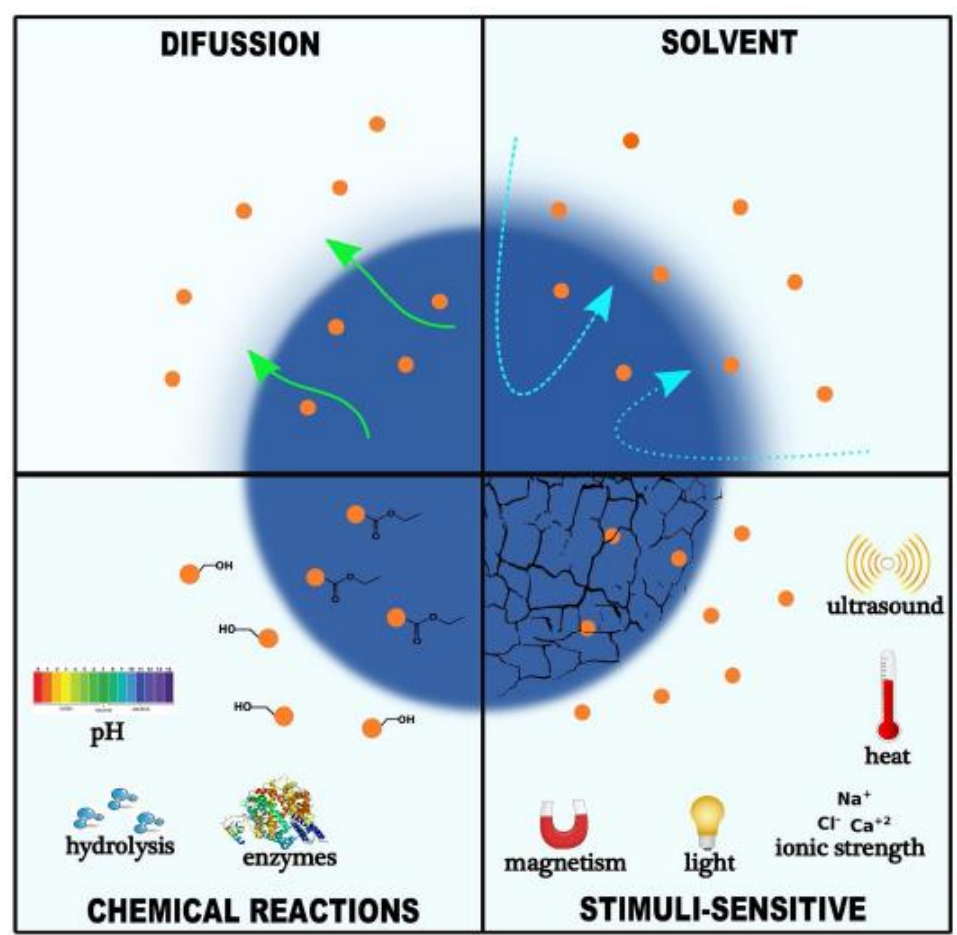

Figure 5: Process of controlled release of drugs [32] 
By using external factors (e.g. heat, light, ultrasound, magnetism [30], pH), stimuli-responsive nanocarriers have exhibited the capability to control the drug release profile, which further may enhance the precision of targeting and allow the better control of dose. To stimulate the controlled release, superparamagnetic iron oxide nanoparticles are mixed with lipids [31] or polymeric nanocarriers using an external magnetic field. In addition, Chen et al. [33] synthesized gold/ ferrous oxide polymer nanoparticles for deployment in chemo-photo-thermal therapy. Such hybrid nanocarriers are one of the most promising tools available currently in nanomedicine as a mixture of different systems having different properties can be engineered into a single system, which improves the diagnostic and therapeutic effectiveness in ever-widening applications.

\subsection{Nanoparticles Used in Drug Delivery}

In drug delivery systems, various bio-polymeric materials are being increasingly utilized. The efficacy of these nanomaterials varies depending on the size, shape and other physical or chemical properties. These materials with their associated characteristics fall into several categories:

Liposomes: This is one of the most studied drug carrier systems which is used in the pharmaceutical industry for the transporatation of different molecules. They were discovered in 1960 by British biophysicist, Alec Bangham. Liposomes are usually of spherical form in the 50$450 \mathrm{~nm}$ size range and composed of steroids and phospholipids [34]. As the membrane structure of liposomes is similar to cell membranes and they allow incorporation of drug in cells, therefore they are considered as a more suitable pharmacokinetic delivery vehicle [34]. It has been proved that liposomes are biodegradable and biocompatible, that they make therapeutic compounds more stable and can be used with hydrophobic and hydrophilic drugs. One significant point about liposomes is that the drugs encapsulated within them are not bio-available until the vesicles are released, hence to increase drug bioavailability, their accumulation in a particular region is important [32].

Polymeric micelles: These are formed when polymeric molecules or amphiphilic surfactants promptly mix in aqueous solution to form a core shell structure. The inner cavity of micelles is hydrophobic (water-hating), which is surrounded by a shell of hydrophilic (water-loving) polymers, like polyethylene glycol (PEG) [32]. These are under $100 \mathrm{~nm}$ in size and have a narrow distribution to avoid fast renal excretion, thus permitting their accumulation through the enhanced permeability and retention (EPR) effect in tumor tissues. Drugs are encapsulated within micelles 
by three different methods i.e. the solvent evaporation process, dialysis process and direct dissolution process. These nanostructures pose strong potential in hydrophobic drug delivery because their interior core structure allows the absorption of drugs which results in enhancement of bioavailability and stability [35].

Dendrimers: Dendrimers are three-dimensional, highly bifurcated and mono-dispersed tree-like structures. The chemical composition and molecular weight of dendrimers can be controlled more precisely in comparison to linear polymers, which makes it comparatively easy to predict their biocompatibility [36]. Dendrimers have been developed for oral, pulmonary, transdermal, ocular (eye) and targeted delivery [37]. There are three different mechanisms through which drug loading is performed in dendrimers: simple encapsulation, covalent conjugation and electro-static interaction.

Inorganic nanoparticles: These nanoparticles include silver, gold, silica etc., which have emerged as a very promising tool in therapeutics since they provide a useful complement to more conventional delivery vehicles. They possess many beneficial characteristics such as easy synthesis, well-established surface functionalization, low toxicity, large surface area and better stability all of which contribute towards providing inorganic nanoparticles with unique features which are ideal for enabling new delivery strategies. Kong et al. [38] have described in detail how drugs can be encapsulated with gold nanoparticle surfaces via covalent bonding or physical absorption and through stimuli-responsive or light activation, they can deliver and control the release of these nano-engineered drugs.

Metallic Nanoparticles: In recent years, the use of metallic nanoparticles has expanded very rapidly in different medical applications including bioimaging, targeted drug delivery, orthopedics, biosensors and hyperthermia [39]. These nanoparticles are particularly useful in thermal therapy and targeting. Conventional methods of heating a tissue by microwave (masers) or laser light, also unfortunately destroy the healthy tissue surrounding the tumor. However targeted magnetic particles constitute a more productive strategy for localized heating of diseased cells. The most studied metallic nanoparticles are silver, copper and gold; however there is a growing interest in the use of alternative metallic nanoparticles such as platium, zinc oxide and titanium oxide [39]. 


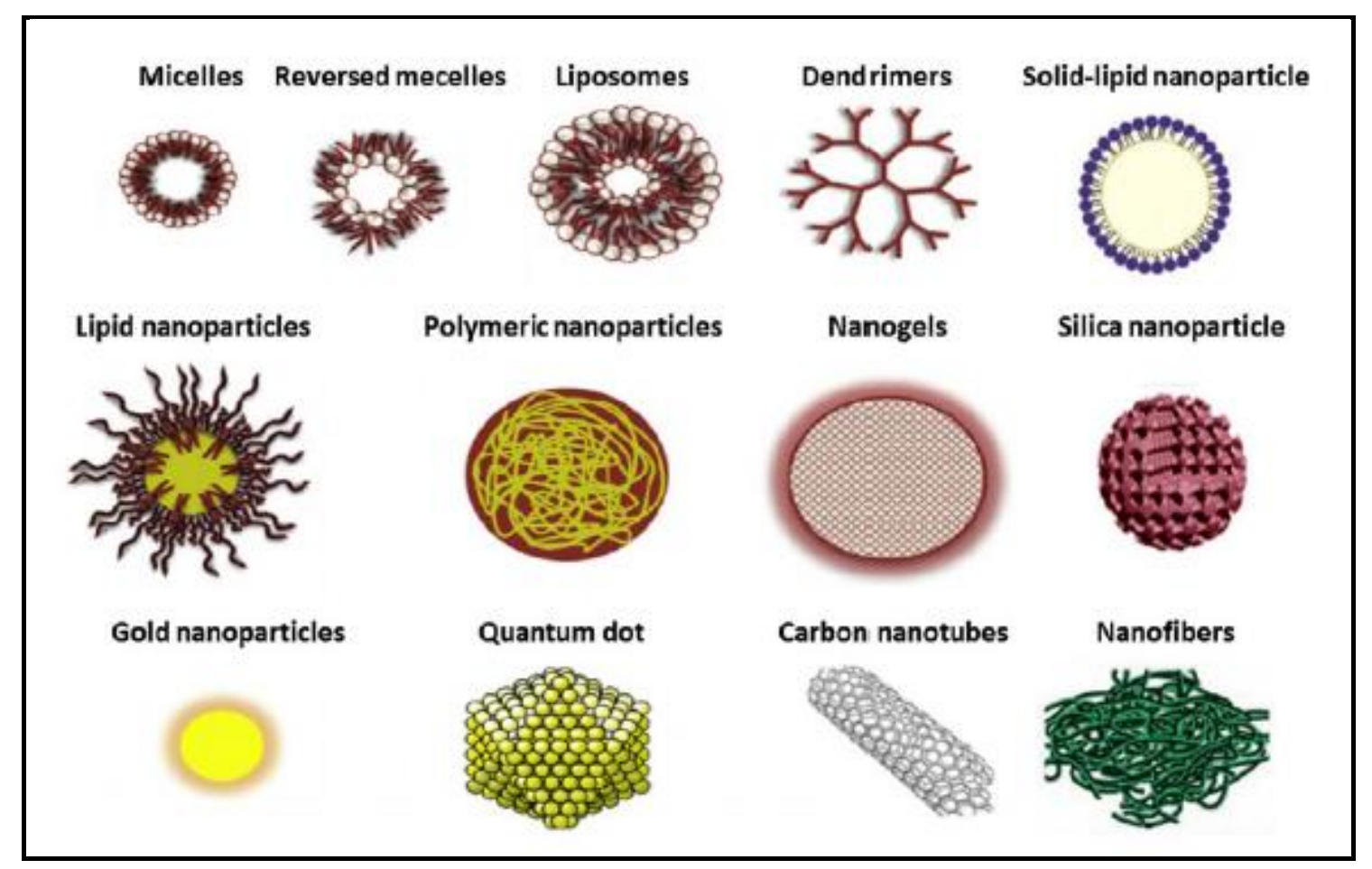

Figure 6 : Types of Nanoparticles [40]

Nanocrystals: Nanocrystals are uniform in size distribution within the $1000 \mathrm{~nm}$ range. They are stabilized by using polymeric surfactants/stabilizers and are $100 \%$ drug without any carrier molecule attached to them and are stable in aqueous dispersions. Nanocrystals are synthesized by top-bottom and bottom-top approaches. Nanocrystals support the absorption of the drug in the human circulatory system by several mechanisms which include enhancement of solubility and suspension rate [41].

Carbon-based Nanomaterials: These have attracted special interest due to their suitability for surface functionalizing in for example the grafting of peptides, proteins and nucleic acids. For drug delivery applications, carbon nanotubes, fullerence and nanodiamonds have been broadly used. Single wall nanotube, multiwall nanotubes and fullerences feature special dimensions, geometry and surface characteristics, which make them applealing for drug carrier delivery.

\subsection{Nano-Magnetic Drug Delivery}

Nowadays, among the most attractive and powerful strategies for pharmacological drug delivery in affected site is the magnetic targeted drug delivery system (MTDDS). In comparison with 
conventional methods, MTDDS are fast-acting and highly efficient and owing to their capacity in reducing toxicity and other adverse side effects, they are becoming more popular in biomedicine [42]. Magnetic materials are of 6 types, among which superparamagnetic materials are the most attractive for magnetic drug delivery. In this targeting, a drug is bound to a magnetic compound and slowly and sytematically introduced into the body, whereupon via an extra-corporeal magnetic field, it is directed to the target site. Depending on the specific application, the nanoparticles are then released from the drug by diffusion or any other mechanism. For designing MTDDS, a variety of factors including magnetic properties, strength of magnetic field, size and drug loading capacity play a major role.

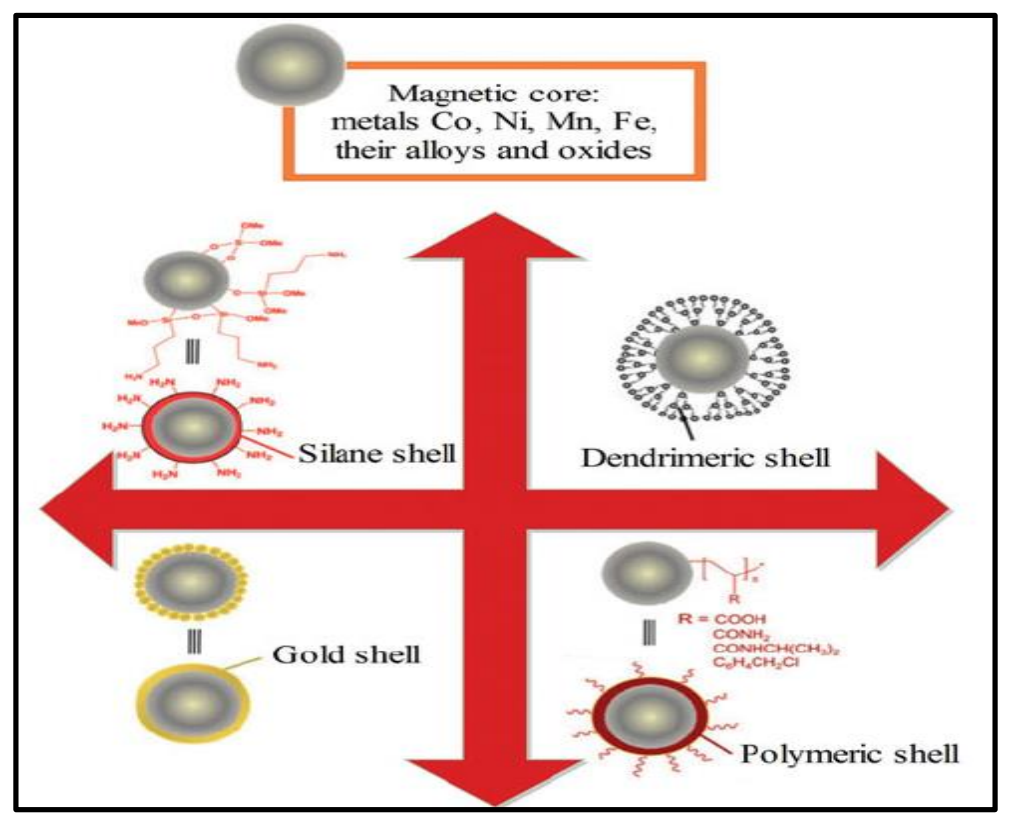

Figure 7 : MNPs with various shells [43]

To improve the efficiency of magnetic targeted drug delivery systems, composite designs of nanoparticles have been explored (hybrid systems). Biomedical applications of magnetic nano particles (MNPs) are classified according to their application: in vivo or in vitro. In vivo applications involve therapeutics and diagnostics while in vitro applications relate to diagnostic usage (seperation/selection). MNPs are widely used in cell and macromolecule seperation, targeted drug delivery, electromagnetic hyperthermia, magnetic resonance imaging (MRI) and gene therapy. However the targeting properties of MNPs make them particularly appropriate for drug delivery systems [44]. Key characteristics of magnetic nanoparticles are that they can be: (a) projected in place by using of magnetic field (b) visualized (c) heated in the presence of magnetic field to generate drug release. To maximize these properties, various functional modification has 
been done in MNPs using polymers, liposomes and silica [45]. To get the best results in drug delivery, MNPs must have peculiar properties such as monodispersity, stability, biocompatibility and superparamagnetism. Superparamagnetism is a necessary property during drug delivery since it is allows the avoidance of agglomeration (a clustering phenomenon which further produces embolisms in blood vessels) [46]. The Food and Drug Administration (FDA) have approved the use of iron oxides (or particular, magnetite) and confirmed that they are totally safe for humans [47].

\section{Nano-based Drug Delivery For Cardovascular Diseases}

According to the World Health Organization (WHO) report given in 2017, cardiovascular diseases (CVDs) are the leading cause of deaths in world. The data shows that 17.7 million deaths occurred up to 2015 and a further 23.6 million are projected to occur up to 2030 [5]. The term cardiovascular disease encompasses, but is not restricted to coronary heart disease, thrombosis and pulmonary embolism etc. All other WHO data corresponds to ischemia and tissue deaths. Heart failure is a clinical disease, having symptoms of fatigue and breathlessness and these symptoms are a result of the reduced quality of cardiac functionality [48]. The current statistics emphasize the urgent requirement for new and more effective therapies and technologies which can prevent these diseases. The existing treatments for CVDs aim to restore the normal blood flow around the damaged vasculature and prevent recurring phenomenon. Banach et al. [49] suggested that by statin therapy, reduction in build-up and thickening of plaques can be achieved. However, such therapies also have adverse side effects and poor patient compliance, which suggests that modifications of such treatments are needed. This among other factors has accelerated the deployment of nanotechnology, (the leading area in $21^{\text {st }}$ century sciences globally), in biomedicine and witnessed the birth of a new field, nanomedicine.

\subsection{Significance}

Nano-based drug delivery systems have great potential in the field of health care. They provide better penetration of drugs through the body as their size allows delivery via injection or other routes. The application of nanoscience in medicine aims to improve the existing treatment regarding these diseases [51]. Nanotechnology provides an effective and safe platform in controlled drug delivery systems for a range of active ingredients, which are directed to lipid 
disorders, angiogenesis within atherosclerosis and inflammation and avoidance of thrombosis among other diseases [52]. Iron oxide super-paramagnetic nanoparticles are a major development in the magnetic drug targeting [53]. The magnetic drug targeted system provides appropriate magnetic gradients, which further increase the concentration of nanoparticles at the affected site [54].

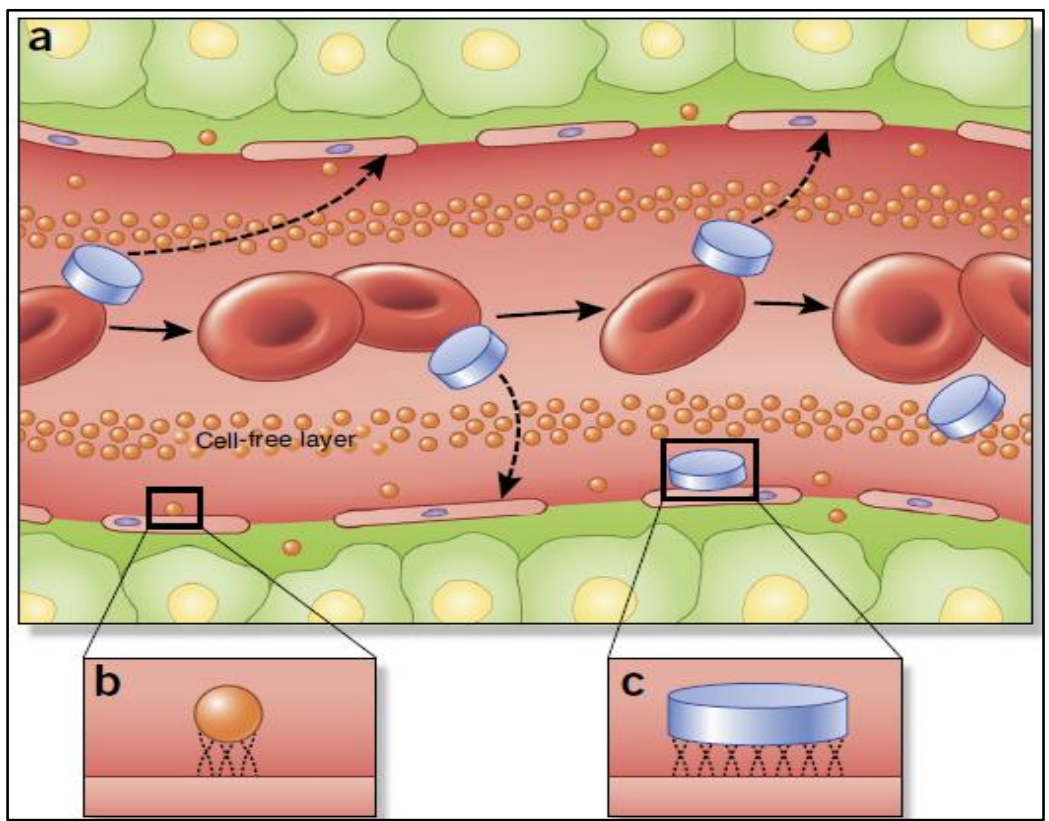

Figure 8: Nanoparticle flow, margination and adhesive properties in blood vessels depending on geometry and particle size [50]

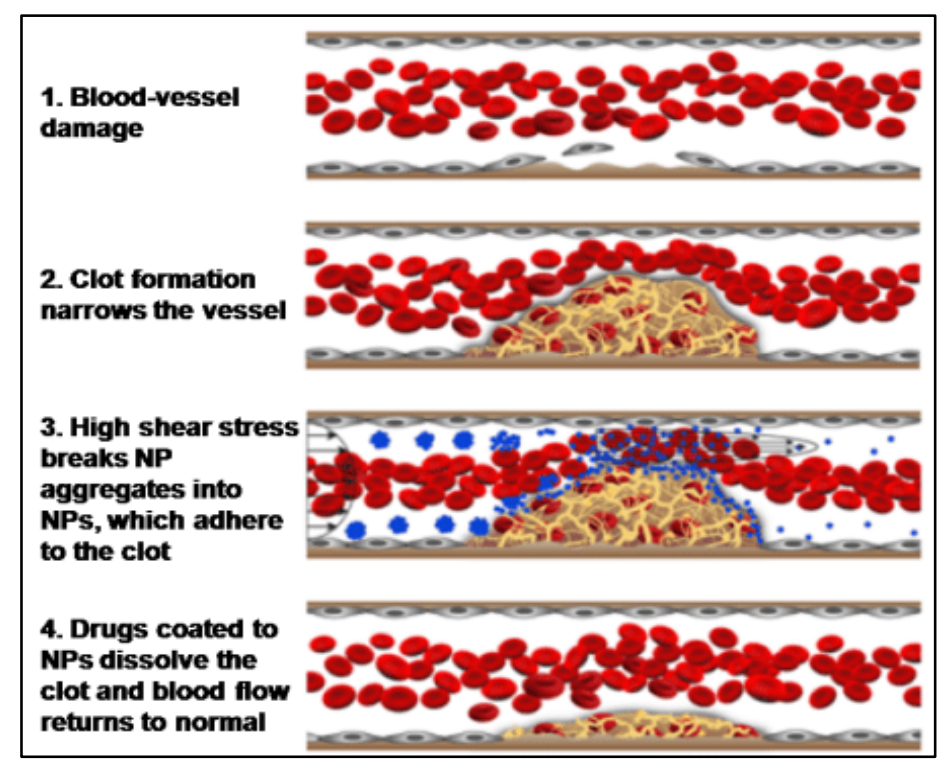

Figure 9: How nanoparticles clear the blood clot [55] 
Nanoparticles can also enhance the delivery of anti-inflammatory agents i.e. glucocorticoids, which further reduce the macrophage accumulation in atherosclerotic lesions. Glucocorticoids have unfavorable profiles such as short half-life times and a large volume of distribution, which results in the necessity for frequent high doses and the associated unbearable side effects such as hypertension and diabetes. To overcome this problem, liposomal formulation is proposed, which prolongs the circulation time, resulting in marked improvement of drug accumulation in the endothelium [56]. For the treatment of atherosclerotic lesions, studies have been conducted using liposomes loaded with phosphatidyl-choline (PC). PC is a compound that increases the concentration of high-density lipoproteins and decreases LDL (low-density lipoprotein) oxidation in atherosclerotic plaque, thus limiting the inflammatory process occurring at this site. In the aortic wall, reduction in plaque volume and cholesterol has been reported after 5 weeks of treatment with liposomal formulation, which suggests that it can become an attractive therapy to reduce atherosclerotic plaques [57].

\subsection{Nano-based Blood Flow (Nano-hemodynamic) Models}

The expansion of nanotechnology has revolutionized biomedicine in the $21^{\text {st }}$ century. Many new nanomaterials have also been implemented in a variety of areas in biomechanics and biofluid mechanics. A sub-set of these nanomaterials (which include carbon nanotubes, nano shells, nanorods and nanowires) is nanofluids, mentioned earlier. Although initially developed for automotive and thermal power applications, nanofluids have been successfully utilized in an astonishing range of clinical applications. These have included both theoretical and experimental studies, largely based on the Buongiorno model [58] which is a two-component nanoscale formulation emphasizing Brownian motion and thermophoretic body force effects (originating in nuclear engineering at MIT) or the Tiwari-Das model [59] which is a volume-fraction-based doping model. Interesting uses of nanofluids in medicine include blood purification systems [60], smart bio-nano-polymer coatings for medical devices [61], nano-drug delivery (pharmacological systems) in cardiovascular treatment $[62,63]$, biodegradable nano-liquids for cerebral pharmacodynamics [64], membrane oxygenator bioreactors [65], orthopaedic lubrication with nano-films (super-lubricated poly (3-sulfopropyl methacrylate potassium salt)-grafted mesoporous silica nanoparticles suspended in starch base liquids) [66], pulsed laser ablation (PLA) ultra-pure silicon nanofluid fabrication for cancer therapy [67], smart biomimetic electro-osmotic nanofluid pumps 
in ocular diagnosis [68], cryopreservation, bone reinforcement via super-paramagnetic nanofluids etc. In hemodynamic therapies, the base liquid is clearly blood and this may be doped with a variety of nanoparticles including gold. It has been discussed earlier in this review that in the field of nanomedicine, nanoparticles have been broadly studied as particular carriers since as drug delivery systems, they deliver excellent results as a result of their biocompatibility, subcellular size, decreased toxicity and sustained release properties. Many theoretical studies have been explored to illustrate the effect of various nanoparticles in blood flows and this area of study is known as nano-hemodynamics. In particular, stenotic nano-hemodynamics has witnessed considerable interest in the engineering sciences and applied mathematics communities owing to its significance in combatting diseased arteries. The many studies reported have also featured numerous computational methods which are required to tackle the nonlinearity of the mathematical models. Nadeem et al. [69] studied the steady blood flow through tapered stenosed arteries using nanoparticles with a Prandtl blood flow mode and a homotopy perturbation method. Akbar and Nadeem [70] explored the influence of nanoparticles in a two-dimensional uniform tube under peristaltic waves with a couple stress rheological model, showing that with increasing thermophoresis and Brownian motion parameters, blood temperature is increased. Ellahi et al. [71] presented a numerical study of composite steady-state stenotic nanofluid hemodynamic flow through vessels with permeable walls. Ahmed et al. [72] investigated the influence of different nanoparticles $\left(\mathrm{Cu}, \mathrm{TiO}^{2}, \mathrm{Al}_{2} \mathrm{O}_{3}\right)$ on Newtonian blood flow in a single stenosed vessel, noting that flow acceleration in the core region is greater for $\mathrm{Al}_{2} \mathrm{O}_{3}$ nanoparticles than either $\mathrm{Cu}$ or $\mathrm{TiO}_{2}$ nanoparticles. Mekheimer et al. [73] considered the simultaneous effect of metallic nanoparticles (copper) and magnetic field on micropolar blood flow through an overlapping mildly stenotic artery, observing that under stronger magnetic field, with higher nanofluid volume fraction, the velocity and wall shear stress are elevated. Nadeem and Ijaz [74] explored the influence of single wall carbon nanotubes (SWCNT) on peristaltic hemodynamic characteristics in multiple stenotic arteries with variable nanofluid viscosity. They found that compared with the pure blood case, the size of the trapping bolus increases with an increment in nanoparticle concentration. Nadeem et. al. [75] studied the impact of different metallic nanoparticles in blood flow through an artery featuring both a stenosis and an aneurysm. Elnaqeeb et al. [76] presented a simulation for blood flow conveying copper nanoparticles through a catheterized single stenotic artery with thrombosis, noting that velocity is increased whereas hemodynamic resistance (impedance) is reduced with 
greater nanoparticle volume fraction. Ahmed et al. [77] addressed the effect of nanoparticle shape factor on Newtonian blood flow in an overlapping (catheterized) stenotic artery for the case of copper nanoparticles, highlighting deviations in hemodynamic behaviour between curved and noncurved arteries. Ijaz et al. [78] studied the influence of hybrid nanoparticles (silver, copper and gold) nanoparticles on blood flow characteristics in a stenotic channel, noting that gold nanoparticles exert a more dramatic impact. Changdar and De [79] have performed an analytical investigation of the effect of gold nanoparticles in magnetohydrodynamics (MHD) blood flow through an irregular shaped stenosed artery. They showed that velocity and displacement profiles exhibit greater fluctuations with smaller nanoparticles. Ponalagusamy et al. [80] described a theoretical viscoplastic blood flow model for unsteady nano-hemodynamics in a tapered single stenotic artery under the influence of magnetic field and periodic body acceleration, for the case of ferric oxide $\left(\mathrm{Fe}_{2} \mathrm{O}_{3}\right)$ nanoparticles. Ijaz et al. [81] investigated the $\mathrm{Cu}$ and $\mathrm{CuO}$ nanoparticlemediated blood flow in an overlapped mildly stenotic artery, noting that wall shear stress and flow resistance decreases with platelet shaped nanoparticles. Ali et al. [82] performed a numerical simulation of time-dependent non-Newtonian (Sisko fluid) nano-pharmacodynamic transport phenomenon in an overlapping tapered artery using Buongiorno's model and a forward time central space (FTCS) method. Results were validated with finite element method (FEM) and it was found that flow rate at stenotic throat decreases with an increase in the Brownian motion parameter. Zaman et al. [83] investigated the unsteady blood flow through a curved stenosed artery with an aneurysm for hybrid nanoparticles (copper, silver) also using the FTCS method. They observed that with greater nanoparticle volume fraction, flow rate, velocity and wall shear stress increases whereas the impedance is reduced. Ahmed et al. [84] considered 6 types of stenosis geometry and studied the effects of magnetohydrodynamic body force and hybrid nanoparticles $\left(\mathrm{Cu}\right.$ and $\left.\mathrm{Al}_{2} \mathrm{O}_{3}\right)$ on electrically conducting micropolar nano-doped arterial blood flow. They noted that among all the stenosis geometries, maximum flow acceleration was computed for the trapezoidal type while maximum deceleration was associated with the irregular symmetric stenosis. Sultan et al. [85] used an Eyring-Powell viscoelastic nanofluid model to simulate time-dependent nanohemodynamic flow in a stenotic, aneurysmic and curved artery, noting that non-Newtonian blood attains higher velocities in the post-stenotic region compared with the stenotic zone. A number of other interesting studies related to application of nanoparticles in pharmacological hemodynamics have been communicated. Very recently, Vasu et al. [86] have developed a robust model of non- 
Newtonian nanofluid hemodynamics with heat and mass diffusion in a stenosed coronary artery in the presence of a radial magnetic field. The study provides a deeper understanding of nano-drug diffusion in the treatment of cardiovascular disease (stenotic arteries). Buongiorno's nanoscale model and the Reiner-Rivlin second order differential model are employed in the formulation. The geometry considered in [86] is reproduced in Fig. 10.

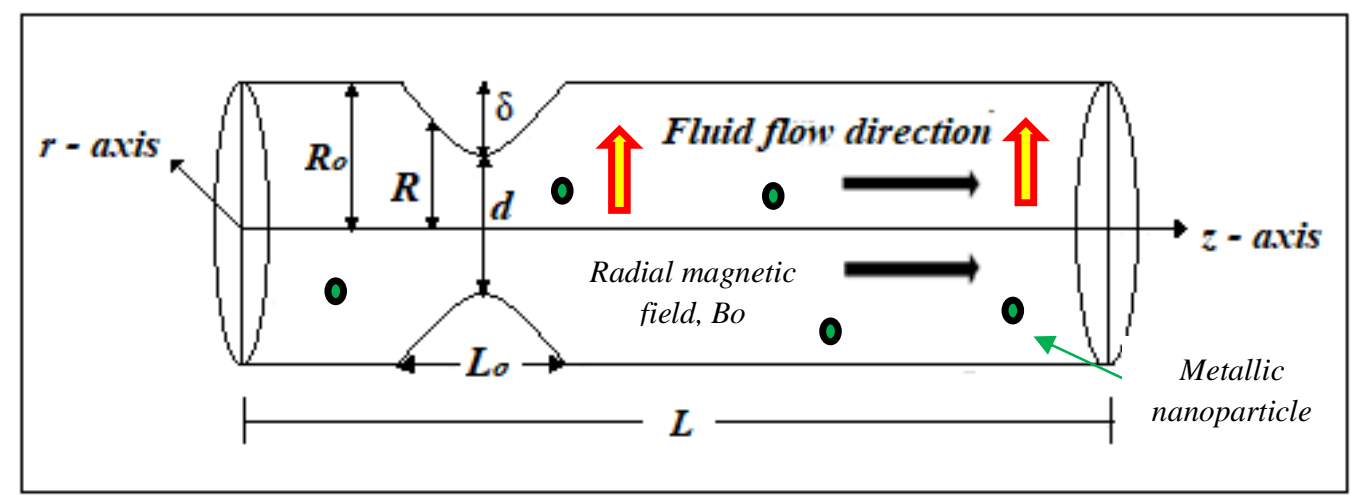

Figure 10. Schematic illustration of stenosed coronary arterial model [86]

The FreeFEM++ finite element code [87] was used to compute hemodynamic characteristics in this study. The finite element mesh was designed with 5928 unstructured fixed triangular elements with 12177 nodes as presented in Figure 11. The mesh generation was accomplished with the automatic FreeFEM++ mesh generator based on the Delaunay-Voronoi algorithm.

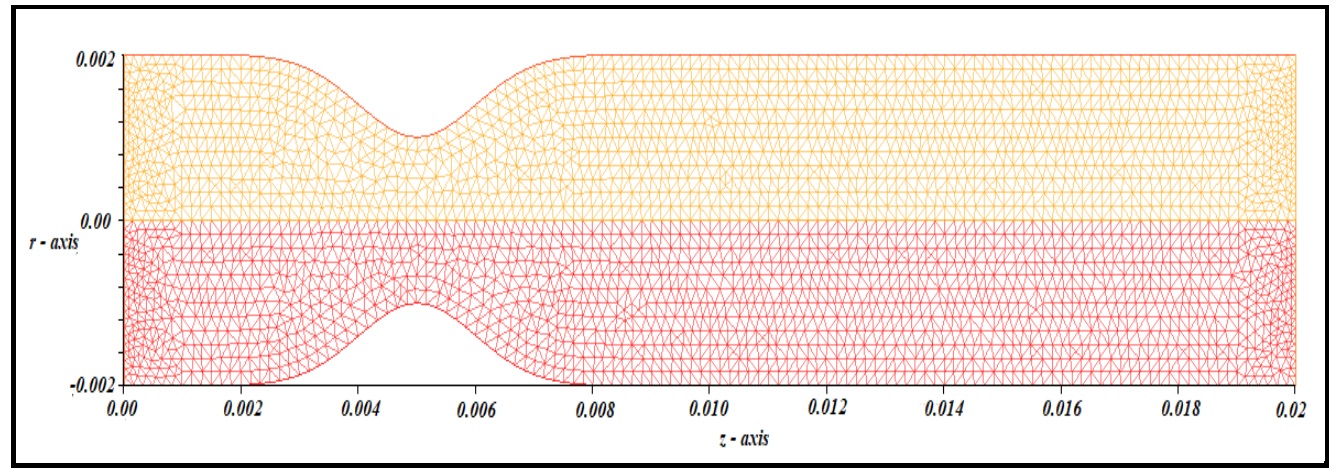

Figure 11. Unstructured triangular fixed finite element mesh [86] 
Some selected simulations from [86] are shown in Figs. 12 a-d for non-dimensional velocity profile for different values of magnetic field parameter $(M)$ and thermophoresis parameter $\left(N_{t}\right)$ is in the arterial domain, corresponding to the mild stenosis case (here the blood flow direction is taken from left to right).

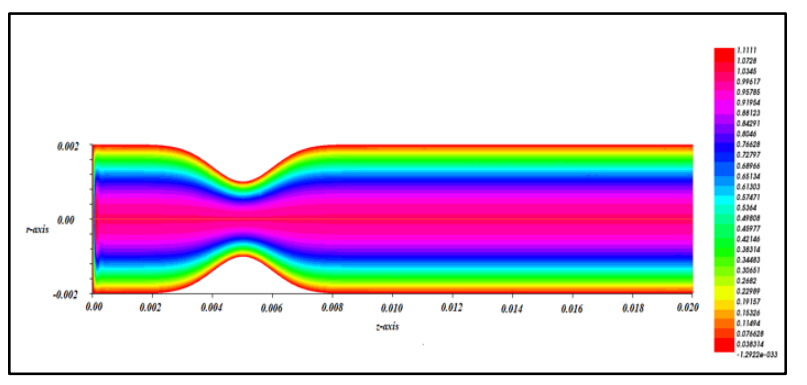

12(a)

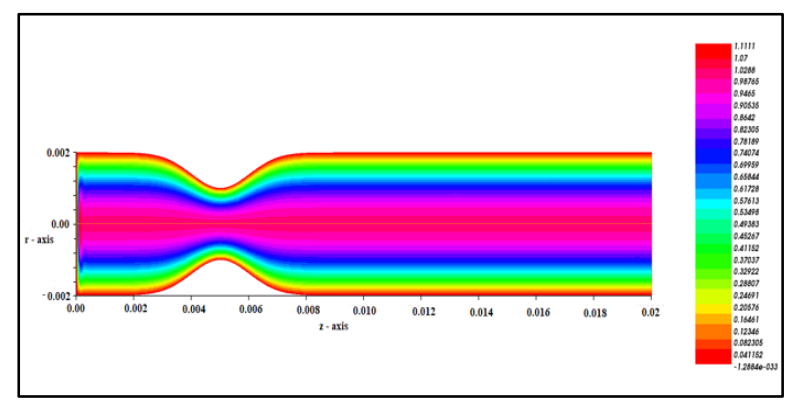

12(c)

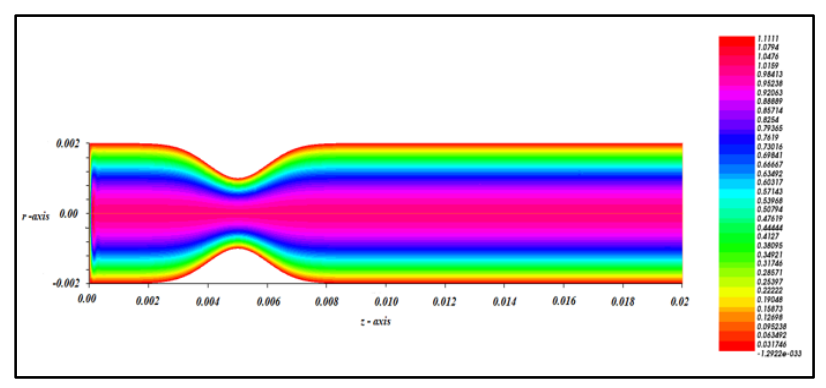

12(b)

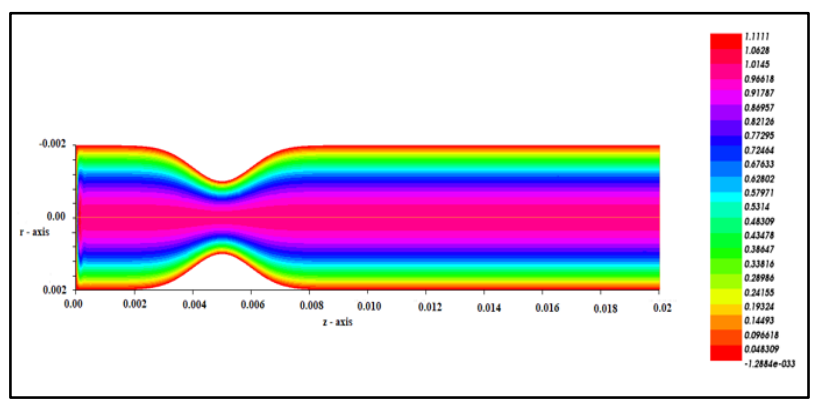

$12(d)$

Figure 12 Velocity field (a) $M=0.3, N_{b}=0.3$ and $N_{t}=0.3$ (b) $M=0.3, N_{b}=0.3$ and $N_{t}=0.6$, (c) $M=0.6, N_{b}=0.3$ and $N_{t}=0.3$, (d) $M=0.6, N_{b}=0.3$ and $N_{t}=0.6$ [86].

Increasing the values of thermophoresis parameter $\left(N_{t}\right)$ from 0.3 to 0.6 (with magnetic parameter, $M$, and Brownian motion parameter, $N_{b}$ fixed) decrease the value of velocity in the vessel. Thermophoresis relates to the migration of nanoparticles under the force of a temperature gradient. With nanoparticles migrating, the momentum is inhibited in the arterial flow and deceleration induced. Effectively thermophoretic body force arises due to the averaged Brownian motion of particles in the nano-doped blood under a steady temperature gradient. Since steady state conditions are assumed, the stronger molecular impulses in the hotter nanofluid region mobilize nanoparticles to migrate towards the colder region, where the molecular impulses are weaker. This destroys momentum in the blood flow and results in a depletion in velocity. In particular, in the core zone strong deceleration is induced. With increasing magnetic parameter $(M)$ from 0.3 to 0.6 as shown in figures $12 \mathrm{a}$ and $12 \mathrm{c}$ (for $N_{t}=0.3$ ) and figures $12 \mathrm{~b}$ and $12 \mathrm{~d}$ (for $N_{t}=0.6$ ) there is also a significant 
decrement in velocity i.e. the core flow is again retarded. The presence of the Lorentzian magnetic drag force resists blood flow and especially generates deceleration in the constricted zone at the stenosis which in turn influences the remainder of the flow domain. In all plots, the velocity is maximum at centreline of the artery and decreases to the boundary at which it vanishes in accordance with the no-slip condition. Computational nano-hemodynamics clearly is a powerful tool for visualization in modern nano-biomedicine and drug delivery systems and provides an excellent methodology for optimizing the impact of different nano-drugs in clinical treatments. Other numerical methodologies are also very promising in this regard and include Lattice Boltzmann [89], molecular dynamics [90], smoothed particle hydrodynamics [91] and boundary element methods [92].

\section{Conclusions}

In the present review, recent advances in nanomedicine have been considered in addition to progress in the delivery of old drugs with new therapeutic methods. Initially, to enhance the solubility, bioavailability, absorption and targeted and controlled release of drugs is the main motivation for implementing nanotechnology in biomedicine. The use of nano- carriers formulated with dendrimers, liposomes, micelles, solid lipid nanoparticles, gold, silver, titanium oxide and cadmium sulphide polymeric nanoparticles together with superparamagnetic iron oxide nanoparticles has been shown to substantially improve the efficacy of conventional pharmacological agents. Magnetic drug delivery systems also offer excellent potential due to their unique properties which are ideal for targeted and controlled delivery. This review has also described significant progress in the use of mathematical and computational hemodynamic models for simulating nanoparticle drug delivery effects in cardiovascular diseases (stenotic arteries, aneurysms etc). Computational nano-hemodynamics offers great advantages in providing deeper insight into the inherent mechanisms involved in nano-drug delivery. Many methods have been developed in this regard and warrant further investigation and corroboration with clinical findings. The advancement in nano-biomedicine along with improved safety and reduced toxicity will be greatly accelerated with computational modelling techniques which are under continuous development by engineers and scientists. 


\section{References}

[1]. Ghassemi, M., \& Shahidian, A. (2017). Nano and bio heat transfer and fluid flow. Academic Press.

[2]. Schlichting H. Boundary Layer Theory. McGraw- Hill Book Company, New York. 1968

[3]. Biswas D. Blood Flow Models: A Comparative Study. Mittal Publications, New Delhi.2000.

[4]. World Health Organization (WHO) (2017) Cardiovascular diseases (CVDs). [Online]. http://www.who.int/mediacentre/factsheets/fs317/en/. Accessed 18 Apr 2018

[5]. Prabhakaran, D., Jeemon, P., Sharma, M., Roth, G. A., Johnson, C., Harikrishnan, S. and Dhaliwal, R. S. (2018). The changing patterns of cardiovascular diseases and their risk factors in the states of India: the Global Burden of Disease Study 1990-2016. The Lancet Global Health, 6(12), e1339-e1351.

[6]. Choi S U S, Eastman J A. Enhancing thermal conductivity of fluids with nanoparticles. ASME Int Mech Eng Congr Exp 1995;66: 99-105

[7]. Kakac S, Pramuanjaroenkij A. Review of convective heat transfer enhancement with nano fluids. Int J Heat Mass Transf 2009; 52: 3187-96.

[8]. Tiwari A K, Ghosh P, Sarkar J, Dahiya H, Parekh J. Numerical investigation of heat transfer and fluid flow in plate heat exchanger using nanofluids. Int J Therm Sci 2014; 85: 93-103.

[9]. Pak BC, Cho YI. Hydrodynamic and heat transfer study of dispersed fluids with submicron metallic oxide particles. Experimental Heat Transfer 1998; 11: 151-70.

[10]. Xuan Y, Roetzel W. Conceptions for heat transfer correlations of nanofluids. International Journal of Heat and Mass Transfer 2000; 43: 3701-7.

[11]. Einstein, A.: Eine neue Bestimmung der Molekul-dimension (A new determination of the molecular dimensions). Annalen der Physik 19(2), 289-306 (1906)

[12]. Brinkman, H. C. The Viscosity of Concentrated Suspensions and Solutions. Journal of Chemical Physics, 20, 571 (1952).

[13]. Hamilton, R. L. and Crosser, O. K. Thermal conductivity of heterogeneous two-component systems. IEC Fundam, 1, 182-191 (1962).

[14]. Mahian, O., Kolsi, L., Amani, M., Estell, P., Ahmadi, G., Kleinstreuer, C., Wongwises, S. (2018). Recent advances in modeling and simulation of nanofluid flows-part I: fundamental and theory. Physics reports. 
[15]. Tawfik, M. M. (2017). Experimental studies of nanofluid thermal conductivity enhancement and applications: A review. Renewable and Sustainable Energy Reviews, 75, 1239-1253.

[16]. Elias F, Christine V. Nanoparticles as drug delivery systems. In: Swarbrick J, editor. Encyclopedia of pharmaceutical science and technology. 4th ed. Taylor \& Francis; 2013.

[17]. Sun, T., Zhang, Y. S., Pang, B., Hyun, D. C., Yang, M., \& Xia, Y. (2014). Engineered nanoparticles for drug delivery in cancer therapy. Angewandte Chemie International Edition, 53(46), 12320-12364.

[18]. Arayne MS, Sultana N, Qureshi F. nanoparticles in delivery of cardiovascular drugs. Pak J Pharm Sci. 2007; 20: 340-8.

[19]. Joseph RR, Venkatraman SS. Drug delivery to the eye: what benefits do nanocarriers offer? Nanomedicine. 2017;12: 683-702.

[20]. Lam P-L, Wong W-Y, Bian Z, Chui C-H, Gambari R. Recent advances in green nanoparticulate systems for drug delivery: efficient delivery and safety concern. Nanomedicine. 2017;12: 357-85.

[21]. Park S-H, Oh S-G, Mun J-Y, Han S-S. Loading of gold nanoparticles inside the DPPC bilayers of liposome and their effects on membrane fluidities. Coll Surf B. 2006; 48:112-8.

[22]. De Villiers, M. M., Aramwit, P., \& Kwon, G. S. (Eds.). (2008). Nanotechnology in drug delivery. Springer Science \& Business Media.

[23]. Kleinstreuer, C., \& Xu, Z. (2018). Computational microfluidics applied to drug delivery in pulmonary and arterial systems. Microfluidics: fundamentals, devices, and applications. Wiley, Weinheim.

[24]. Lu, H., Wang, J., Wang, T., Zhong, J., Bao, Y., \& Hao, H. (2016). Recent progress on nanostructures for drug delivery applications. Journal of Nanomaterials, 2016, 20.

[25]. Mignani, S., El Kazzouli, S., Bousmina, M., \& Majoral, J. P. (2013). Expand classical drug administration ways by emerging routes using dendrimer drug delivery systems: a concise overview. Advanced drug delivery reviews, 65(10), 1316-1330.

[26]. Wong, P. T., \& Choi, S. K. (2015). Mechanisms of drug release in nanotherapeutic delivery systems. Chemical reviews, 115(9), 3388-3432.

[27]. Siepmann, F., Herrmann, S., Winter, G., \& Siepmann, J. (2008). A novel mathematical model quantifying drug release from lipid implants. Journal of Controlled Release, 128(3), 233-240. 
[28]. Ding, C., \& Li, Z. (2017). A review of drug release mechanisms from nanocarrier systems. Materials Science and Engineering: C, 76, 1440-1453.

[29]. Lee, J. H., \& Yeo, Y. (2015). Controlled drug release from pharmaceutical nanocarriers. Chemical engineering science, 125, 75-84.

[30]. Hervault, A., \& Thanh, N. T. K. (2014). Magnetic nanoparticle-based therapeutic agents for thermo-chemotherapy treatment of cancer. Nanoscale, 6(20), 11553-11573.

[31]. Alonso, J., Khurshid, H., Devkota, J., Nemati, Z., Khadka, N. K., Srikanth, H., \& Phan, M. H. (2016). Superparamagnetic nanoparticles encapsulated in lipid vesicles for advanced magnetic hyperthermia and biodetection. Journal of Applied Physics, 119(8), 083904.

[32]. Patra, J. K., Das, G., Fraceto, L. F., Campos, E. V. R., del Pilar Rodriguez-Torres, M., Acosta-Torres, L. S., ... \& Habtemariam, S. (2018). Nano based drug delivery systems: recent developments and future prospects. Journal of nanobiotechnology, 16(1), 71.

[33]. Chen, C. W., Syu, W. J., Huang, T. C., Lee, Y. C., Hsiao, J. K., Huang, K. Y., ... \& Lai, P. S. (2017). Encapsulation of $\mathrm{Au} / \mathrm{Fe} 3 \mathrm{O} 4$ nanoparticles into a polymer nanoarchitecture with combined near infrared-triggered chemo-photothermal therapy based on intracellular secondary protein understanding. Journal of Materials Chemistry B, 5(29), 5774-5782.

[34]. Bozzuto, G., \& Molinari, A. (2015). Liposomes as nanomedical devices. International journal of nanomedicine, 10, 975.

[35]. Xu, W., Ling, P., \& Zhang, T. (2013). Polymeric micelles, a promising drug delivery system to enhance bioavailability of poorly water-soluble drugs. Journal of drug delivery, 2013.

[36]. Zhu, J., \& Shi, X. (2013). Dendrimer-based nanodevices for targeted drug delivery applications. Journal of Materials Chemistry B, 1(34), 4199-4211.

[37]. Kesharwani, P., Jain, K., \& Jain, N. K. (2014). Dendrimer as nanocarrier for drug delivery. Progress in Polymer Science, 39(2), 268-307.

[38]. Kong, F. Y., Zhang, J. W., Li, R. F., Wang, Z. X., Wang, W. J., \& Wang, W. (2017). Unique roles of gold nanoparticles in drug delivery, targeting and imaging applications. Molecules, 22(9), 1445.

[39]. McNamara, K., \& Tofail, S. A. (2015). Nanosystems: the use of nanoalloys, metallic, bimetallic, and magnetic nanoparticles in biomedical applications. Physical Chemistry Chemical Physics, 17(42), 27981-27995. 
[40]. Martín Giménez, V. M., Kassuha, D. E., \& Manucha, W. (2017). Nanomedicine applied to cardiovascular diseases: latest developments. Therapeutic advances in cardiovascular disease, 11(4), 133-142.

[41]. Junyaprasert VB, Morakul B. Nanocrystals for enhancement of oral bioavailability of poorly water-soluble drugs. Asian J Pharm Sci. 2015;10:13-23.

[42]. Cao Q, Han X, Li L. Enhancement of the efficiency of magnetic targeting for drug delivery: development and evaluation of magnet system. J Magn Magn Mater. 2011;323:1919-1924.

[43]. Assa, F., Jafarizadeh-Malmiri, H., Ajamein, H., Vaghari, H., Anarjan, N., Ahmadi, O., \& Berenjian, A. (2017). Chitosan magnetic nanoparticles for drug delivery systems. Critical reviews in biotechnology, 37(4), 492-509.

[44]. Nguyen TKT, Leduc T, Ian R. Biomedical applications: magnetic nanoparticles. In: Lyshevski SE, editor. Dekker encyclopedia of nanoscience and nanotechnology. $3^{\text {rd }}$ ed. Taylor \& Francis \& CRC Press; 2014. p. 1740-1743.

[45]. Wankhede, M., Bouras, A., Kaluzova, M., \& Hadjipanayis, C. G. (2012). Magnetic nanoparticles: an emerging technology for malignant brain tumor imaging and therapy. Expert review of clinical pharmacology, 5(2), 173-186.

[46]. Kim J-E, Shin J-Y, Cho M-H. Magnetic nanoparticles: an update of application for drug delivery and possible toxic effects. Arch Toxicol. 2012;86:685-700.

[47]. Obermeier A, Kuchler S, Matl FD, et al. Magnetic drug targeting as new therapeutic option for the treatment of biomaterial infections. J Biomater Sci Polym Ed. 2012;23:2321-2336.

[48]. Ponikowski, P., Voors, A. A., Anker, S. D., Bueno, H., Cleland, J. G., Coats, A. J., ... \& Jessup, M. (2016). 2016 ESC Guidelines for the diagnosis and treatment of acute and chronic heart failure: The Task Force for the diagnosis and treatment of acute and chronic heart failure of the European Society of Cardiology (ESC). Developed with the special contribution of the Heart Failure Association (HFA) of the ESC. European journal of heart failure, 18(8), 891-975.

[49]. Banach, M., Serban, C., Sahebkar, A., Mikhailidis, D. P., Ursoniu, S., Ray, K. K., ... \& Garcia-Garcia, H. M. (2015). Impact of statin therapy on coronary plaque composition: a systematic review and meta-analysis of virtual histology intravascular ultrasound studies. BMC medicine, 13(1), 229. 
[50]. Blanco E, Shen H, Ferrari M. Principles of nanoparticle design for overcoming biological barriers to drug delivery. Nature Biotechnology volume 33, pages 941-951 (2015).

[51]. Radomska, A., Leszczyszyn, J., \& Radomski, M. W. (2016). The nanopharmacology and nanotoxicology of nanomaterials: new opportunities and challenges. Adv Clin Exp Med, 25(1), 151-162.

[52]. Rhee, J. W., \& Wu, J. C. (2013). Advances in nanotechnology for the management of coronary artery disease. Trends in cardiovascular medicine, 23(2), 39-45.

[53]. Janko, C., Dürr, S., Munoz, L.E., Magnetic drug targeting reduces the chemotherapeutic burden on circulating leukocytes. Int J Mol Sci 2013; 14: 7341-7355.

[54]. Bietenbeck, M., Florian, A., Faber, C., Sechtem, U., \& Yilmaz, A. (2016). Remote magnetic targeting of iron oxide nanoparticles for cardiovascular diagnosis and therapeutic drug delivery: where are we now? International journal of nanomedicine, 11, 3191.

[55]. Kimlicka L., Nanoparticles that clear blood clot- Shear activated nanotherapeutics, https://www.somethingaboutscience.com/?p=722, July 2014.

[56]. Lobatto, M. E., Fayad, Z. A., Silvera, S., Vucic, E., Calcagno, C., Mani, V., ... \& Metselaar, J. M. (2010). Multimodal clinical imaging to longitudinally assess a nanomedical antiinflammatory treatment in experimental atherosclerosis. Molecular pharmaceutics, 7(6), 2020-2029.

[57]. Ruiz-Esparza, G. U., Flores-Arredondo, J. H., Segura-Ibarra, V., Torre-Amione, G., Ferrari, M., Blanco, E., \& Serda, R. E. (2013). The physiology of cardiovascular disease and innovative liposomal platforms for therapy. International journal of nanomedicine, 8, 629.

[58]. Buongiorno, J., Convective transport in nanofluids. ASME J. Heat Transfer, 128, 3: 240$250,2006$.

[59]. Das, S.K., S.U. Choi, W. Yu and T. Pradeep, Nanofluids: Science and Technology, CRC Press, USA, 416pp, 2007.

[60]. O. Anwar Bég, M.M. Rashidi, M. Akbari, A. Hosseini, Comparative numerical study of single-phase and two-phase models for bio-nanofluid transport phenomena, J. Mechanics in Medicine and Biology, 14, 1450011.1-31, 2014.

[61]. M. J. Uddin, M.N. Kabir, O. Anwar Bég and Y. Alginahi Chebyshev collocation computation of magneto-bioconvection nanofluid flow over a wedge with multiple slips 
and magnetic induction, Proc. IMechE: Part N-Journal of Nanomaterials, Nanoengineering and Nanosystems (2018). doi: 10.1177/2397791418809795 (15 pages).

[62]. S.A.A. Rizvi and A.M. Saleh, Applications of nanoparticle systems in drug delivery technology, Saudi Pharmaceutical J., 26(1): 64-70, 2018.

[63]. P. H. da Costa Santos, Development of a biodegradable nanofluid for brain drug delivery, Master Science in Biomedical Engineering, University of Coimbra, Portugal, September (2015).

[64]. N. S. Akbar, D. Tripathi and O. Anwar Bég, Variable-viscosity thermal hemodynamic slip flow conveying nanoparticles through a permeable-walled composite stenosed artery, European Physical Journal Plus, 132: 294 -305, 2017.

[65]. O. Anwar Bég, Nonlinear multi-physical laminar nanofluid bioconvection flows: Models and computation, A. Sohail, Z. Li (Eds.): Computational Approaches in Biomedical NanoEngineering, Wiley, Chapter 5, pp. 113-145 (2018).

[66]. Y. Yan et al., Euryale ferox seed-inspired super-lubricated nanoparticles for treatment of osteoarthritis, Adv. Functional Materials, 29 (4), 1807559, 2019.

[67]. M.M. Raj Sha et al., Ultra-pure silicon nanofluid by laser ablation: thermal diffusivity studies using thermal lens technique, Applied Physics B, 124: 213, 2018.

[68]. D. Tripathi, A. Sharma and O. Anwar Bég, Electrothermal transport of nanofluids via peristaltic pumping in a finite micro-channel: effects of Joule heating and HelmholtzSmoluchowski velocity, Int. J. Heat Mass Transfer, 111, 138-149, 2017.

[69]. Nadeem, S., Ijaz, S., \& Akbar, N. S. (2013). Nanoparticle analysis for blood flow of Prandtl fluid model with stenosis. International Nano Letters, 3(1), 35.

[70]. Akbar, N. S., \& Nadeem, S. (2013). Intestinal flow of a couple stress nanofluid in arteries. IEEE Transactions on Nanobioscience, 12(4), 332-339.

[71]. Ellahi, R., Rahman, S. U., Nadeem, S., Akbar, N. S. (2014). Blood flow of nanofluid through an artery with composite stenosis and permeable walls. Applied Nanoscience, 4(8), 919-926.

[72]. Ahmed, A., Nadeem, S. (2016). The study of $\left(\mathrm{Cu}, \mathrm{TiO}_{2} ; \mathrm{Al}_{2} \mathrm{O}_{3}\right)$ nanoparticles as antimicrobials of blood flow through diseased arteries. Journal of Molecular Liquids, 216, 615-623. 
[73]. Mekheimer, K. S., Elnaqeeb, T., El Kot, M. A., Alghamdi, F. (2016). Simultaneous effect of magnetic field and metallic nanoparticles on a micropolar fluid through an overlapping stenotic artery: Blood flow model. Physics Essays, 29(2), 272-283.

[74]. Nadeem, S., Ijaz, S. (2015). Single wall carbon nanotube (SWCNT) examination on blood flow through a multiple stenosed artery with variable nanofluid viscosity. AIP Advances, 5(10), 107217.

[75]. Nadeem, S., Ijaz, S. (2015). Influence of metallic nanoparticles on blood flow through arteries having both stenosis and aneurysm. IEEE Transactions on Nanobioscience, 14(6), 668-679.

[76]. Elnaqeeb, T., Mekheimer, K. S., Alghamdi, F. (2016). Cu-blood flow model through a catheterized mild stenotic artery with a thrombosis. Mathematical Biosciences, 282, 135146.

[77]. Ahmed, A., Nadeem, S. (2017). Shape effect of Cu-nanoparticles in unsteady flow through curved artery with catheterized stenosis. Results in Physics, 7, 677-689.

[78]. Ijaz, S., Nadeem, S. (2017). A biomedical solicitation examination of nanoparticles as drug agents to minimize the hemodynamics of a stenotic channel. The European Physical Journal Plus, 132(11), 448.

[79]. Changdar, S., De, S. (2019). Analytical investigation of nanoparticle as a drug carrier suspended in a MHD blood flowing through an irregular shape stenosed artery. Iranian Journal of Science and Technology, Transactions A: Science, 43(3), 1259-1272.

[80]. Ponalagusamy, R., Priyadharshini, S. (2017). Numerical modelling on pulsatile flow of Casson nanofluid through an inclined artery with stenosis and tapering under the influence of magnetic field and periodic body acceleration. Korea-Australia Rheology Journal, 29(4), 303-316.

[81]. Ijaz, S., Iqbal, Z., Maraj, E. N., Nadeem, S(2018). Investigation of Cu-CuO/blood mediated transportation in stenosed artery with unique features for theoretical outcomes of hemodynamics. Journal of Molecular Liquids, 254, 421-432.

[82]. Ali, N., Zaman A., Sajid M., Bég, Anwar O., Shamshuddin M. D. and Ali Kadir (2018), Numerical simulation of time-dependent non-Newtonian nano-pharmacodynamic transport phenomena in a tapered overlapping stenosed artery, Nanoscience and Technology: An International Journal, 9, 247282. 
[83]. Zaman, A., Ali, N., Ali, I. (2018). Effects of nanoparticles (Cu (Copper), Silver (Ag)) and slip on unsteady blood flow through a curved stenosed channel with aneurysm. Thermal Science and Engineering Progress, 5, 482-491.

[84]. Ahmed, A., Nadeem, S. (2017). Effects of magnetohydrodynamics and hybrid nanoparticles on a micropolar fluid with 6-types of stenosis. Results in Physics, 7, 4130-4139.

[85]. Sultan F., Khan N. A., Qasim M., Afridi, M. I. (2019). Numerical simulation of the flow of nano-Eyring-Powell fluid through a curved artery with time-variant stenosis and aneurysm. Nihon Reoroji Gakkaishi, 47(2), 75-85.

[86]. Vasu, B., Ankita Dubey and O. Anwar Bég (2019). Finite element analysis of non-Newtonian magnetohemodynamic flow conveying nanoparticles through a stenosed coronary artery. Heat Transfer-Asian Research. https://doi.org/10.1002/htj.21598

[87] Hecht, F., New development in FreeFEM++, J. Numerical Mathematics, 20, 251-266, 2012. [88] https://www.verywellhealth.com/what-is-atherosclerosis-1745908

[89] C. Sun and L.L. Munn (2008), Lattice Boltzmann simulation of blood flow in digitized vessel networks, Comput Math Appl. 55(7): 1594-1600.

[90] S. Safaei et al., Molecular dynamics simulations of Janus nanoparticles in a fluid flow, Soft Matter, $15,6742-6752$.

[91] M. Al-Saad et al. (2016). Blood flow simulation using smoothed particle hydrodynamics,

Proc. $24^{\text {th }}$ UK Conference of the Association for Computational Mechanics in Engineering 31 March - 1 April, Cardiff University, Cardiff, UK.

[92] Peng H.M., Yang D.Q., Yang X.Y. (2007) The boundary element analysis of bloodstream in the bifurcation deformity blood vessel. In: Computational Mechanics. Springer, Berlin, Heidelberg, Germany. 